\title{
Boldina disminuye la apoptosis miocárdica post isquemia reperfusión en la rata.
}

René López 1, 4, Marlene Arismendi, Juan Carlos Sáez³, Iván Godoy², María Paz Ocaranza .

\author{
1) Laboratorio Cardiología Molecular, \\ 2) Laboratorio Cardiología no Invasiva, División de Enfermedades \\ Cardiovasculares, Facultad de Medicina \\ 3) Departamento de Ciencias Fisiológicas, Facultad de Ciencias Biológicas, \\ 4) Interno Medicina Pontificia Universidad Católica de Chile. \\ Financiamiento: PUC Biomedicina BM09/2007
}

\section{Resumen:}

Antecedentes: En la isquemia/reperfusión (IR) miocárdica es relevante la pérdida de cardiomiocitos por apoptosis. En estos, los hemicanales (HC) permiten el ingreso de sustancias proapoptóticas durante la IR. Boldina (B), compuesto extraído del Peumus boldus, ha demostrado ser antioxidante y bloquear los $\mathrm{HC}$.

Objetivo: Determinar el efecto de boldina sobre la apoptosis de cardiomiocitos de ratas sometidas a IR. Métodos: Ratas macho de $200 \mathrm{~g}$ de peso se sometieron a ligadura reversible de la arteria coronaria izquierda por 30 minutos (I) y posterior reperfusión (R) por 24 horas post I. Un subgrupo de estos animales recibió una dosis de boldina intraventricular (IR+B, $40 \mathrm{mg} / \mathrm{Kg}$ ) y luego dos dosis vía gavage $(75 \mathrm{mg} / \mathrm{Kg}$ ) a los 30 y 60 minutos post-R. Como controles se usaron ratas sham con operación ficticia, que recibieron igual tratamiento. Se determinaron las masas corporal (MC) y cardiaca relativa (MCR) y presión arterial sistólica (PAS). Porcentaje de cardiomiocitos apoptóticos (CMAP), otras células apoptóticas (OCAP) y total de células apoptóticas (TCAP) se determinó por TUNEL. La activación de metaloproteinasas (MMPs) 2 y 9 se determinó por zimografía y el mRNA de MCP-1 por RT-PCR.

Resultados: La boldina no modificó la MC y la MCR. Sin embargo, disminuyó significativamente la PAS así como el \%CMAP y el \%TCAP en el grupo IR+B versus IR (CMAP $69 \pm 1,5$ vs $44 \pm 0,4$, $\mathrm{p}=0,016$, TCAP $71 \pm 2,4$ vs $57 \pm 1,5, \mathrm{p}=0,016$ ). No se encontraron diferencias en el OCAP, actividad de MMPs y en los niveles de mRNA de MCP-1.

Conclusiones: Boldina disminuyó la PAS y la apoptosis de cardiomiocitos post IR. Su efecto no es mediado por modificaciones en la actividad de MMPs y expresión génica de MCP-1.

Correspondencia:

María Paz Ocaranza,

Departamento de Enfermedades Cardiovasculares.

Facultad de Medicina. P. Universidad Católica de Chile.

Tel: +562-3543407, Fax: +562-6321924.

E-mail: mocaran@med.puc.cl 


\section{Boldine decreases post ischemia / reperfusion myocardial apoptosis in rats}

Background: Ischemia / reperfusion (IR) is relevant in the myocardial loss of cardiomyocytes through apoptosis. During IR, hemi channels (HC) allow the entry of proapoptotic substances to the cell. Boldine, a compound extracted from Peumus boldus, has proven to be antioxidant and to block HC.

Objective: To determine the effect of boldine on cardiomyocyte apoptosis in rats subjected to IR.

Methods: Male rats, body weight (BW) $200 \mathrm{~g}$, were subjected to reversible ligation of the left coronary artery for 30 minutes (I) and subsequent reperfusion (R) for 24 hours. A subset of these animals (IR+B) received an intraventricular dose of boldine $(40 \mathrm{mg} / \mathrm{kg})$ and then two doses via gavage $(75 \mathrm{mg} / \mathrm{kg})$ at 30 and $60 \mathrm{minu}-$ tes post-R. Sham operated rats $(\mathrm{S})$ receiving the same treatment were used as controls. We determined body weight (BW), relative heart mass (RHM) and systolic

\section{Introducción}

El infarto al miocardio (IAM) constituye una de las principales causas de insuficiencia cardíaca (IC) ${ }^{1}$. En el último tiempo se han generado grandes avances en el tratamiento del IAM enfocados en la reperfusión del área comprometida ${ }^{2}$, lo cual ha reducido la mortalidad en el período agudo post $\mathrm{IAM}^{3}$. En la isquemia con reperfusión el estrés oxidativo deteriora la función cardíaca por daño oxidativo de membranas y macromoléculas celulares, produciendo disfunción y muerte celular mediante procesos de apoptosis o necrosis en el miocardio ${ }^{4}$. La apoptosis, a diferencia de la necrosis, está presente en etapas tempranas y tardías post IAM, ubicándose los cardiomiocitos apoptóticos tanto en el miocardio viable o remoto como en la zona adyacente al IAM $^{5}$

El estrés oxidativo se produce por un desbalance entre la producción de especies reactivas tanto del oxígeno (ROS) como del nitrógeno (RNS) y los mecanismos antioxidantes, en su mayoría enzimáticos. En fibroblastos cardíacos las ROS han mostrado activar metaloproteasas (MMPs) de la matriz extracelular ${ }^{6}$. Adicionalmente, los ROS pueden modular la actividad de diversas moléculas y señales de transducción intracelular, tales como las proteínas involucradas en el proceso de excitación-contracción, canales iónicos, canales liberadores de calcio del retículo sarcoplásmico y miofilamentos ${ }^{7}$.

Los canales de unión en hendidura (CUH) comunican el citoplasma de células adyacentes y los hemicanales blood pressure (SBP). Percentage of apoptotic cardiomyocytes (CMAP), other apoptotic cells (OCAP) and total apoptotic cells (TCAP) were determined by TUNEL. Activation of metalloproteinases (MMPs) 2 and 9 was determined by zymography and MCP-1 mRNA levels by RT-PCR.

Results: Compared to IR alone, IR+Boldine did not change BW or RCM, but significantly decreased PAS, $\operatorname{TCAP}(71 \pm 2.4$ vs $57 \pm 1.5, \mathrm{p}=0.016)$ and CMAP $(69 \pm$ 1.5 vs $44 \pm 0.4, \mathrm{p}=0.016)$. No difference was observed in the OCAP, MMPs activity and MCP-1 mRNA levels. Conclusions: Boldine decreased SBP and post-IR cardiomyocyte apoptosis without effect on other cells. This effect was not mediated by MMPs activity or MCP-1 gene expression.

Key words: Boldine, apoptosis, ischemia/reperfusion.

(HCs) comunican el medio intra con el extra celular. Se ha demostrado que los CUH formados por conexina 43 se nitrosilan y esta modificación covalente se asocia a la apertura de los $\mathrm{HCs}$ presentes en la superficie celular y por lo tanto a un aumento de la muerte celular ${ }^{8}$. En general, es aceptado que los HCs permanecen cerrados la mayor parte del tiempo (baja probabilidad de apertura) y son permeables a iones y moléculas pequeñas incluyendo algunas que juegan un papel importante en la señalización intercelular paracrina, como el calcio, ATP y prostaglandina E2 ${ }^{9}$.

La boldina ((S)-(1,10-dimetoxi-2,9 dihidroxiaporfina) es un compuesto extraído del boldo (Peumus boldus), árbol endémico de Chile. Las propiedades del boldo pueden resumirse como: estimulante de la digestión, sedante sobre el sistema nervioso, citoprotector y anti-inflamatorio en modelos experimentales ${ }^{10}$. En estudios in vitro, la boldina a concentraciones entre 10-5 10-4 M, ha mostrado ejercer un efecto relajante de la musculatura lisa sobre ileum de rata y una pequeña parte es mediada por acción anticolinérgica. Se ha propuesto que este alcaloide bloquea receptores $\alpha$ adrenérgicos y es antagonistas de canales de calcio en ratas ${ }^{11}$. También, presenta buena afinidad por los receptores de dopamina tipo D1 y D2. Resultados preliminares en células HeLa transfectadas con conexina43, la principal conexina del miocardio, revelan que boldina y glaucina-un derivado de la boldina- actúan como inhibidores de HCs y no afectan el estado funcional de los $\mathrm{CUH}^{12}$.

A la fecha no existen antecedentes en la literatura respecto de los efectos de la boldina en la función ventricular, 
remodelamiento miocárdico ni muerte celular en modelos experimentales de isquemia con reperfusión miocárdica. El objetivo de este estudio fue determinar el efecto de boldina sobre la apoptosis de cardiomiocitos de ratas sometidas a isquemia con reperfusión y su efecto en la actividad de MMPs y niveles de mRNA de MCP-1 miocárdico.

\section{Métodos}

La presente investigación se realizó de acuerdo a la "Guía para el cuidado y uso de animales de laboratorio" publicado por el "National Health Institute" (NIH No 85-23, 1985) y fue aprobada por la Comisión de Investigación de la Facultad de Medicina de la P. U. Católica de Chile. Durante el período de estudio, los animales se mantuvieron en el Vivero del Laboratorio de Cardiología Molecular en el Centro de Investigaciones Médicas de la Facultad de Medicina y recibieron agua y comida ad libitum en condiciones controladas de luz ( 12 horas de ciclos de luz/oscuridad), temperatura ambiental de $21^{\circ} \mathrm{C}$, humedad ambiental de $50 \%$, ventilación adecuada y libre de ruidos.

\section{Modelo experimental.}

Se usaron ratas macho Sprague Dawley de $200 \pm 10 \mathrm{~g}$ de peso. La isquemia se indujo por oclusión reversible de la rama descendente anterior de la arteria coronaria izquierda por 30 minutos y posteriormente la arteria se reperfundió por 24 horas (IR). Las ratas IR fueron randomizadas para recibir 1 minuto antes de la reperfusión suero fisiológico o boldina (40 $\mathrm{mg} / \mathrm{kg}$ peso vía intraventricular) y posteriormente, 30 y 90 min post inicio de la reperfusión $(75 \mathrm{mg} /$ $\mathrm{kg}$ peso, vía gavage) ${ }^{13}$. Como grupo control (Sham), se usaron animales sometidos a operación ficticia y tratados con boldina según el protocolo. Los animales fueron eutanasiados a las 24 horas post inicio de la reperfusión.

\section{Estudios hemodinámicos y funcionales}

La presión arterial sistólica (PAS) se midió por el método pletismográfico en la cola de los animales bajo suave anestesia con éter etílico un vez por semana por investigadores ciegos al tratamiento ${ }^{14}$. La función del ventrículo izquierdo se evaluó por ecocardiografía transtorácica bidimensional al término del tratamiento, para lo cual se usó un transductor sectorial electrónico de ultrabanda S12 Philips con rango de frecuencia de operación de 5-12 $\mathrm{MHz}$ 2D, doppler color, doppler pulsado y continuo, adaptable al ecocardiógrafo Sonos 5000. Para la evaluación de la función ventricular los animales IR y Sham fueron previamente anestesiados con Ketamina ( $35 \mathrm{mg} / \mathrm{Kg}$ peso) y Xilacina $(7 \mathrm{mg} / \mathrm{Kg}$ peso) y su tórax fue rasurado. Se determinó el diámetro de fin diástole (DFDVI) y de sístole (DFSVI) del ventrículo izquierdo (VI), la fracción de acortamiento del VI (FA) y el grosor de la pared anterior y posterior del VI (GPAVI, GPPVI) ${ }^{14}$.

\section{Evaluación de la hipertrofia ventricular izquierda.}

El grado de hipertrofia cardíaca (HC) se cuantificó por la relación entre la masa ventricular (MV) y la masa corporal $(\mathrm{MC}, \mathrm{MV} / 100 \mathrm{~g} \mathrm{PC})^{14}$.

4. Determinación de apoptosis por ensayo TUNEL (terminal transferase-mediated dUTP nick labeling)

Para la identificación de células apoptóticas presentes en el miocardio, cortes histológicos embebidos en parafina de $5 \mu \mathrm{m}$ de grosor, se tiñeron mediante la técnica TUNEL ${ }^{15}$, utilizando para ello un kit comercial (In Situ Cell Death Detection Kit, POD, Roche). El procedimiento se aplicó de acuerdo a las recomendaciones del fabricante con un tiempo de incubación de 60 minutos a $37^{\circ} \mathrm{C}$ para la reacción de la transferasa y pretratando las muestras por calentamiento en horno de microondas por 5 minutos con citrato de sodio $0,1 \mathrm{M}$. Los cortes se contratiñeron con hematoxilina. La conversión de la señal se realizó con peroxidasa de rábano y diaminobenzidina para luego tomar microfotografías en microscopio de luz a un aumento $20 \mathrm{X}$ (Eclipse E-800, Nikon) y cámara digital (Coolpix 4500, Nikon) a 0,05 mm2/campo de visión ${ }^{16}$.

Para cada corte se analizaron 16 campos de visión consistentes a lo largo de la circunferencia del miocardio. En cada campo se contó el número de núcleos marcados positivamente (TUNEL(+)) diferenciando aquellos núcleos ubicados claramente en el centro del citoplasma de un cardiomiocito. También se determinó el número total de núcleos de cardiomiocitos visibles para calcular el porcentaje de cardiomiocitos TUNEL(+) por campo de visión. Los núcleos fueron considerados como cardiomiocitos cada vez que estos estaban situados en el centro de una célula de citoplasma grande con morfología característica de cardiomiocito en corte transversal y límites nítidos. El procedimiento fue realizado por un observador ciego a la intervención.

\section{Niveles de de mRNA de la proteína quimiotractante de macrófagos tipo 1 (MCP-1) de ventrículo izquierdo} Se siguió el procedimiento descrito por Ocaranza et al14. Brevemente, el RNA total del ápice del VI se aisló por el método del trizol y se cuantificó por espectroscopia a $260 \mathrm{~nm} / 280 \mathrm{~nm}$. El cDNA se obtuvo por transcripción reversa a partir de 1,5 $\mu \mathrm{g}$ de RNA total tratado con DNAsa. Los ensayos de PCR se realizaron usando los siguientes protocolos de amplificación y secuencias de partidores, 38 ciclos de $1 \mathrm{~min}$ a $92^{\circ} \mathrm{C}, 1 \mathrm{~min}$ a $53^{\circ} \mathrm{C}, 1 \min$ a $72^{\circ} \mathrm{C}$ y pos- 
teriormente 10 min a $72{ }^{\circ} \mathrm{C}$. Las secuencias nucleotídicas de los partidores sentido y antisentido fueron $5^{\prime}$-CAGGTCTCTGTCACGCTTCT-3’ y 5’-GTGCTTCAGGTGGTTGTGG-3`, respectivamente ${ }^{17}$. La intensidad de las bandas se cuantificó por densitometría y se normalizó con respecto a la banda $18 \mathrm{~S}$ del RNA ribosomal.

\section{Evaluación de la actividad de metaloproteinasa} (MMP) 2 y 9 por zimografía.

Se utilizó la técnica descrita por Petterson et al.18, de zimografía en geles de gelatina. Para ello, trozos de VI viable de $100 \mathrm{mg}$ se homogenizaron en tampón de lisis (PBS, $0,5 \%$ deoxicolato de sodio, $0,1 \%$ SDS, $1 \mathrm{mM}$ ortovanadato de sodio, $1 \mathrm{mM}$ PMSF, $1 \% \mathrm{M}$, Nonidet P-40, 1,5 $\mu \mathrm{M}$ aprotinina, $21 \mu \mathrm{M}$ leupeptina y $14,6 \mu \mathrm{M}$ pepstatina A) y centrifugados por 10 minutos a $7.000 \mathrm{G}$ a $4^{\circ} \mathrm{C}$ para separar restos celulares ${ }^{18}$. La concentración de proteínas del sobrenadante se midió por el método de Bradford18. Se utilizaron $100 \mu \mathrm{g}$ de proteína diluida en amortiguador no reductor SDS 2x (126 mM Tris- $\mathrm{HCl}, 20 \%$ glicerol, $4 \%$ SDS, $0,005 \%$ azul de bromofenol, $\mathrm{pH} 6,8)$. Las muestras se cargaron en geles SDS-PAGE al $8 \%$ con $0,1 \%$ de gelatina y corridas a $100 \mathrm{mV}$ a temperatura ambiente. Posteriormente, el gel se lavó dos veces con 2,5 \% Tritón X-100 durante 30 minutos a temperatura ambiente, e incubado a $37^{\circ}$ por 18 horas con $50 \mathrm{mM}$ de HEPES, pH 7,5, 0,2 $\mathrm{M} \mathrm{NaCl}, 5 \mathrm{mM} \mathrm{CaCl}$ y $20 \mu \mathrm{mol} / \mathrm{L}$ de $\mathrm{ZnCl}$. Finalmente los geles se tiñeron con azul de coomassie $(0,2 \%$ azul de coomasie, $10 \%$ ácido acético glacial, 50\% metanol). La actividad se determinó mediante análisis densitométrico mediante un scanner Hewlewt-Packard y el software image-j de análisis densitométrico.

\section{Análisis estadístico y presentación de resultados}

Las comparaciones se realizaron utilizando el test no paramétrico Mann-Whitney. Las diferencias significativas se consideraron con valor $\mathrm{p}<0,05$ (con 2 colas de 0,025 cada una). Los resultados se expresaron como promedio \pm SEM.

\section{Resultados}

1 Parámetros morfométricos y hemodinámicas y función ventricular izquierda.

La tabla 1 muestra los parámetros hemodinámicos, destacando que a las dosis de boldina usadas sólo se observó una disminución significativa de la presión arterial sistólica en las ratas IR+boldina respecto a ratas con Sham+Boldina y ratas IR $(78 \pm 4$ vs $95 \pm 4$ y $97 \pm 4$, $\mathrm{p}=0,006$, respectivamente). No se encontraron diferencias en la masa corporal (MC) ni masa cardíaca relativa (MCR, mg de corazón/masa corporal), como tampoco se produjo edema pulmonar ya que no se observaron cambios en la masa pulmonar relativa (MPR).

Las variables ecocardiográficas evaluadas se muestran en la tabla 2. A las 24 horas post cirugía no se observaron diferencias entre los grupos experimentales.

\begin{tabular}{|c|c|c|c|}
\hline & Sham+boldina & IR & IR+boldina \\
\hline N & 8 & 10 & 10 \\
\hline MC (g) & $190 \pm 4$ & $201 \pm 3$ & $198 \pm 4$ \\
\hline PAS $(\mathrm{mmHg})$ & $95 \pm 4^{*}$ & $97 \pm 4^{*}$ & $78 \pm 4$ \\
\hline MCR & $412 \pm 19$ & $423 \pm 13$ & $423 \pm 14$ \\
\hline MPR & $816 \pm 65$ & $607 \pm 64$ & $616 \pm 96$ \\
\hline
\end{tabular}

Los valores representan promedio \pm SEM. Abreviaciones: IR: isquemia reperfusión; $M C$ : masa corporal; PAS: presión arterial sistólica; $M C R$. masa cardíaca relativa (mg corazón*100/MC), MPR: masa pulmonar relativa (mg pulmón*100/MC). *p=0.006 vs IR+boldina

\begin{tabular}{|c|c|c|}
\hline & IR & IR+boldina \\
\hline $\mathbf{N}$ & 10 & 10 \\
\hline DFDVI (mm) & $7,43 \pm 0,16$ & $6,98 \pm 0,32$ \\
\hline DFSVI (mm) & $5,38 \pm 0,44$ & $5,21 \pm 0,49$ \\
\hline AFDVI (mm2) & $4,12 \pm 0,16$ & $4,18 \pm 0,29$ \\
\hline AFSVI (mm2) & $2,11 \pm 0,29$ & $2,22 \pm 0,30$ \\
\hline AFS/AFD & $0,49 \pm 0,06$ & $0,47 \pm 0,06$ \\
\hline DPPVI (mm) & $1,29 \pm 0,02$ & $1,23 \pm 0,04$ \\
\hline DPAVI (mm) & $1,13 \pm 0,08$ & $1,13 \pm 0,07$ \\
\hline
\end{tabular}

Valores representan promedio \pm SEM. Abreviaciones: DFDVI: diámetro de fin de diástole del ventrículo izquierdo; DFSDVI. diámetro de fin de sístole del VI; AFDVI: área de fin de diástole del VI; AFSVI: área de fin de sístole del VI; DPP: diámetro de la pared posterior del VI; DPAVI: diámetro de la pared anterior del VI.

\section{Apoptosis}

La administración temprana de boldina al inicio de la isquemia y durante la reperfusión disminuyó la apoptosis de los cardiomiocitos en un $35 \%$ y un $19,7 \%$ en las células totales positivas para la reacción de TUNEL, respecto al grupo IR sin tratamiento. Las células no cardiomiocíticas positivas para TUNEL no presentaron diferencias estadísticamente significativas entre ambos grupos experimentales (figura 1A y 1B). 
Figura 1: Detección de células apoptóticas por ensayo TUNEL medidas 24 horas después de la isquemia en los grupos experimentales.
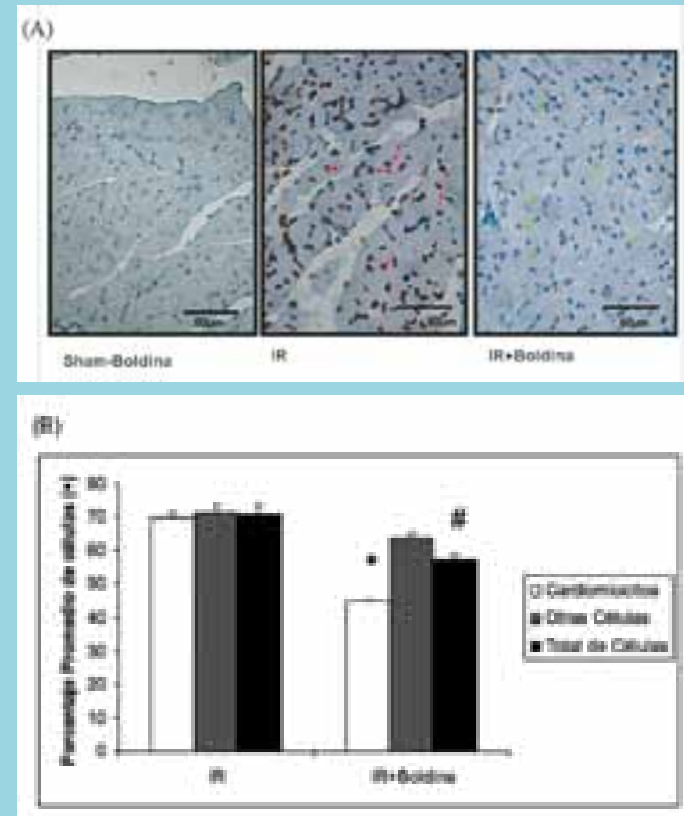

A) Imágenes obtenidas de una rata con IR y una IR+boldina. Flechas rojas: células TUNEL(+) con morfología de cardiomiocito. Flechas verdes núcleos de cardiomiocitos negativos a TUNEL. B) Porcentaje promedio de núcleos de positivos a TUNEL. $N=5$ por grupo, * $p<0,0001$ vs $I R, \# p=0,03$ respecto a $I R$.

\section{Niveles de mRNA de MCP-1}

Se utilizó la expresión génica de MCP-1 para ver si es que las diferencias en la apoptosis encontradas por TUNEL podrían ser explicadas o al menos asociadas a una disminución concomitante en la inflamación. El tejido empleado para esto fue miocardio del ápice ventricular debido a que no se dispuso de miocardio de una zona más cercana a la base, que es la zona de donde se obtuvo el tejido para la realización de TUNEL. La figura 2A muestra los resultados del RT-PCR de algunas de las muestras evaluadas. No se encontraron diferencias significativas entre el grupo IR+boldina vs las ratas con IR, p=0,24 (Figura 2B).

\section{Actividad de MMP-2 y MMP-9}

Las MMP-2 y 9 se asocian a remodelado ventricular post infarto al miocardio. La MMP-9 se activa en etapas tempranas mientras que la MMP-2 se activa en fases tardías post isquemia miocárdica. El aumento en la actividad de MMP-9 post isquemia se ha asociado a apoptosis en el miocardio. Para determinar si la disminución en la apoptosis encontrada en el TUNEL del grupo IR+boldina respecto del grupo IR se asocia a una disminución en la actividad de MMPs de matriz extracelular, se realizó un ensayo de zimografía18. La figura 3A representa los resultados de las zimografías de las ratas IR vs IR+B. No se encontraron diferencias significativas en las actividades de MMP-2 y MMP-9 ( $\mathrm{p}=0,755$ y $\mathrm{p}=0,662$ respectivamente), en los grupos experimentales evaluados (figura 3B).

Figura 2: Efecto de boldina en los niveles de mRNA de MCP-1 en ventrículo izquierdo alejado de la isquemia.

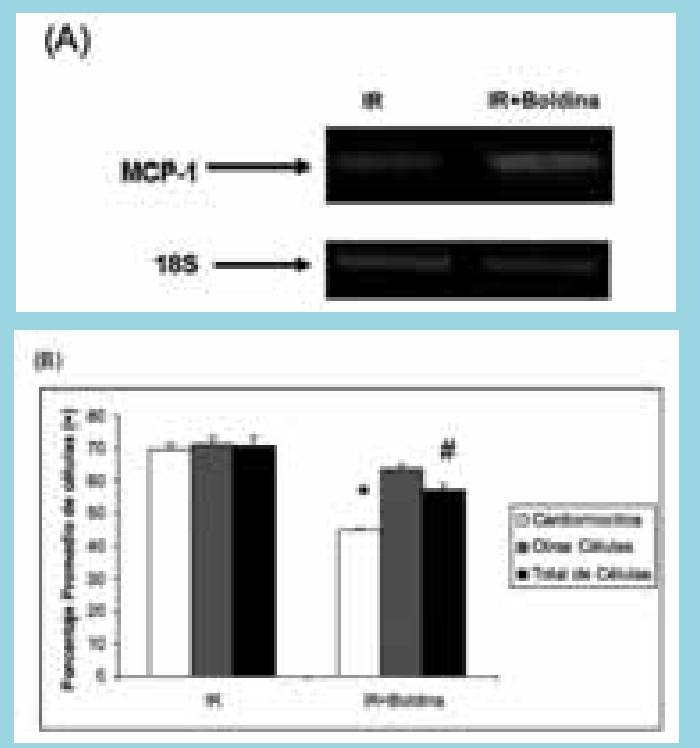

A) RT-PCR de MCP-1, B) Niveles de MCP-1 expresado en veces respecto a al grupo experimental IR. $N=6$ por grupo.

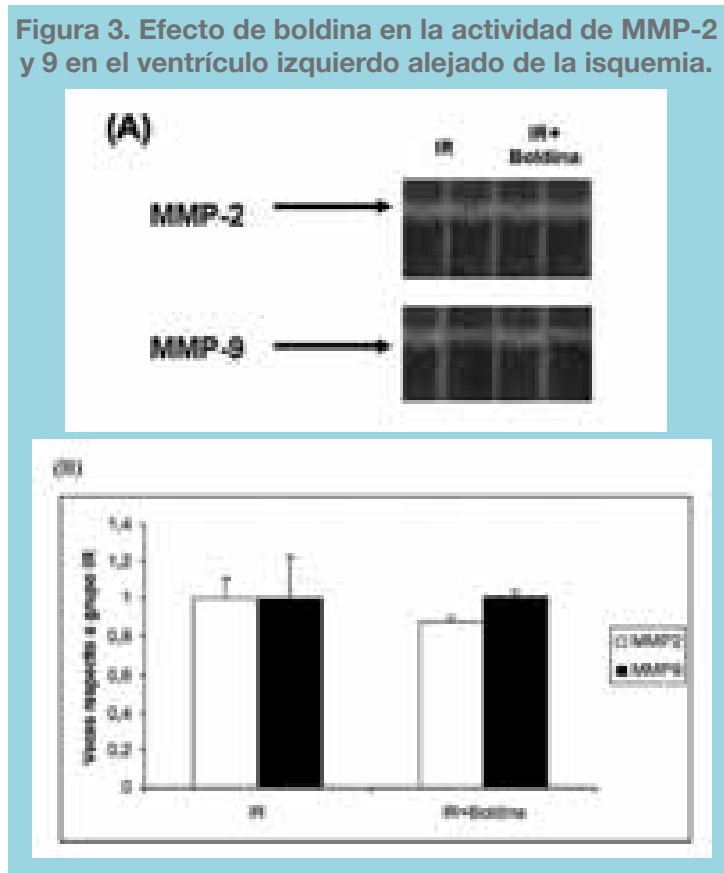

(A) Zimografías de MMPs 2 y 9. (B) Actividad de MMP-2 y MMP-9 expresada en veces respecto a grupo IR. $N=8$ por grupo. 


\section{Discusión}

Los principales resultados de este trabajo mostraron que la administración de boldina: 1) disminuyó tempranamente la PAS y la apoptosis de cardiomiocitos y del total de células miocárdicas, post isquemia. 2) El efecto antiapoptótico de boldina no es mediado por la disminución de la expresión del factor proinflamatorio MCP-1 y tampoco, por la disminución en la actividad de MMPs-2 y 9.

El daño miocárdico por IR se caracteriza por la aparición temprana de procesos de necrosis e inflamación, seguida de apoptosis tanto de miocardiocitos como de otras células del miocardio. Estos procesos tienen tal relevancia que solamente 30-60 minutos de IR compromete el miocardio en una extensión comparable a la observada normalmente luego de 24 horas de oclusión permanente ${ }^{30}$. Nuestros resultados muestran claramente el aumento significativo de la apoptosis de cardiomiocitos del grupo IR respecto al grupo Sham, producto de la activación de procesos celulares que se gatillan en este tipo de daño.

Entre los mecanismos iniciales de daño por IR se han descrito la acidificación celular durante el período de isquemia que se revierte rápidamente durante la reperfusión, llevando a la acumulación de sodio intracelular a través del intercambiador $\mathrm{Na}-\mathrm{H}$ y Na-HCO $3^{31}$. El aumento del sodio intracelular lleva a un aumento del flujo de calcio desde el retículo sarcoplásmico hacia el sarcolema mediado por el intercambiador $\mathrm{Na}-\mathrm{Ca}$ sin posibilidad de reincorporarlo ${ }^{32}$. Esta sobrecarga de calcio produce un estado de hipercontractibilidad de la fibra miocárdica ${ }^{33}$, depleción de ATP ${ }^{34}$, daño ultraestructural de mitocondrias y aturdimiento miocárdico ${ }^{35}$. La IR genera aumento en la producción de especies reactivas del oxígeno (ROS) en la mitocondria, llevando a la apertura de poros mitocondriales permitiendo el paso de las ROS al citoplasma ${ }^{36}$, generando nuevas ROS vía xantina oxidasa y culminando con la formación del apoptosoma ${ }^{37}$.

Considerando la relevancia del estrés oxidativo característico de este daño es que se han realizado esfuerzos por identificar sustancias con capacidad de reducir los radicales libres o disminuir su efecto sobre el tejido afectado ${ }^{3}$. Boldina es una molécula con conocidas propiedades antioxidantes ${ }^{20}$. Al respecto, se ha descrito en células HeLa transfectadas con conexina ${ }^{43}$, la principal conexina del miocardio, la boldina actúa inhibiendo los HCs sin afectar el estado funcional de los $\mathrm{CUH}^{12}$. Además de sus características antioxidantes teóricas derivadas de su estructura molecular, se ha demostrado in vitro que boldina tiene un efecto protector ante el daño oxidativo mediado por catecolaminas en mitocondrias de neuronas dopaminérgicas ${ }^{21}$.
Igualmente, en cortes de hipocampo privados de oxígeno y glucosa para simular el daño producido por isquemia, se demostró que boldina aumentó significativamente la viabilidad celular ${ }^{22}$. También, se ha descrito un efecto protector en hepatocitos aislados sometidos a estrés oxidativo ${ }^{23}$. En modelos in vivo la boldina disminuyó el daño hepático post exposición a tetracloruro de carbono ${ }^{23}$ y a cisplatino ${ }^{24}$, demostrando esto último un efecto quimioprotector. Además, se ha descrito que boldina reduce significativamente el edema inflamatorio ${ }^{25}$. Al respecto, en un modelo murino de enfermedad inflamatoria intestinal, el pretratamiento con boldina diminuyó la actividad mieloperoxidasa sugiriendo una menor infiltración de neutrófilos ${ }^{26}$. En términos de lipoxidación boldina diminuyó las lesiones ateroscleróticas en aorta sin alterar los niveles de LDL, HDL y triglicéridos, lo que sugiere que el efecto observado sería mediado por su efecto antioxidante ${ }^{28}$. En un modelo murino de diabetes en que se exponen a estreptozotocina (STZ) la cual, por medio de radicales libres mitocondriales, daña las células beta pancreáticas por estrés oxidativo, boldina previno el aumento de la glucosa plasmática y la pérdida de peso generada por la $\mathrm{STZ}^{27}$.

Tanto por experimentos in vivo como in vitro, boldina ha demostrado propiedades antioxidantes, las cuales estarían dando cuenta de su efecto en la prevención de la apoptosis por IR. Nuestros resultados muestran claramente y por primera vez que boldina disminuyó la apoptosis de los cardiomiocitos, efecto farmacológico que se suma a los descritos en otros tejidos.

Es conocido que durante la isquemia se genera un intenso estímulo para la liberación de óxido nítrico a nivel endotelial con efecto favorable mediado por vasodilatación asociado a un efecto sobre la agregación plaquetaria, adhesión de leucocitos y captación de radicales libres ${ }^{38}$. Durante la reperfusión se genera un medio saturado de ROS que genera peroxinitrito que afecta a distintas sustancias vasoactivas y además activa al sistema inmune a través de un aumento en quimioquinas, citoquinas y moléculas de adhesión. Las MMPs habitualmente se activan por proteólisis del propéptido originalmente sintetizado ${ }^{40}$, no obstante, se ha observado que en condiciones de estrés oxidativo con aumento de peroxinitrito libre se produce una activación directa no proteolítica de la MMP-2 y MMP-9. A su vez, la activación de estas MMPs se ha asociado a liberación de citocromo $\mathrm{C}$, aumento en la actividad de caspasa 3 y de la expresión de la vía Bax teniendo como resultado la apoptosis. Nuestros resultados muestran que el efecto antiapoptótico de la boldina no es mediado por cambios en la actividad de MMPs 2 y 9 . Al respecto no se puede descartar que la participación de las MMPs pudiera gati- 
llarse en etapas posteriores y no en etapa temprana como la evaluada en este estudio.

El estrés oxidativo y radicales peroxinitrilo aumentan la permeabilidad endotelial, inflamación, producción de citoquinas y quimiotractantes con expresión de "toll like receptors" y activación de la cascada del complemento ${ }^{39}$. Se ha observado que en el daño por IR, MCP-1 aumenta su expresión. La sobreexpresión de MCP-1 en el miocardio genera infiltración por monocitos/macrófagos y vasculopatía arteriolar trombótica oclusiva, la que a su vez se asocia a isquemia, fibrosis intersticial y dilatación ventricular. Además, MCP-1 induce la expresión de la proteína inducida por MCP (MCPIP) la que a su vez se ha identificado como un nuevo factor de transcripción cuya expresión causa apoptosis. En nuestro modelo experimental y a los tiempos evaluados no se observó la participación de MCP-1 como mediador del efecto de la boldina. Estos resultados pueden ser producto que los cambios en la expresión de genes ocurren tempranamente, es decir antes de las 24 horas, respecto en este caso al cambio en el número de células apoptóticas.

Boldina es un compuesto con efecto descrito en receptores alfa adrenérgicos ${ }^{19}$, dopaminérgicos ${ }^{29}$, canales de calcio $^{11}$ y receptores colinérgicos ${ }^{11}$. Además se ha observado un efecto vasodilatador en vasculatura renal ${ }^{19}$. Nosotros observamos que en condiciones de IR+Boldina la PAS fue significativamente menor comparado con los grupos Sham+Boldina e IR. Este efecto podría estar en relación con los descritos y/o potenciado por los eventos patoló- gicos característicos de esta injuria, sin embargo, debe ser considerado en el contexto de una evaluación temprana cuya evolución se desconoce.

Constituyen limitantes de este estudio el esquema de administración de boldina que es nuevo, por lo que se desconoce exactamente a que concentraciones de boldina disponibles en miocardio se logran los efectos observados. No se realizó un estudio de toxicidad, si bien se trabajó con dosis divulgadas en la literatura, la vía de administración intraventricular de boldina es nueva, siendo interesante contar con datos de esta índole. La característica temporal del modelo resulta limitante al intentar explorar posibles mecanismos de acción de boldina que den cuenta de los resultados.

Este es el primer trabajo que muestra un efecto de boldina sobre un modelo de daño miocárdico, siendo relevante tanto por la prevalencia de la enfermedad cardiovascular isquémica, como también por el original efecto de este compuesto. Considerando que boldina es un extracto obtenido desde una especie endémica de Chile, la apertura de cada vez nuevos usos farmacológicos ofrece importantes oportunidades en el desarrollo e implementación de formulaciones que a futuro tengan un rol terapéutico en humanos.

En conclusión Boldina es un compuesto que en las dosis administradas, en modelos tempranos post IR en ratas, tiene un efecto hipotensor y disminuye significativamente la apoptosis de cardiomiocitos, sin alterar la expresión génica de MCP-1 ni la actividad de MMP-2 y MMP-9.

\section{Referencias:}

1.- KANNEL WB. Vital epidemiologic clues in heart failure. J Clin Epidemiol. 2000;53:229-35.

2.- DIAZ L, FAJADET J, CASSAGNEAU B, ROBERT G, MAR-

$\mathrm{CO} \mathrm{J}$. The short-term results and angiographic predictors of subacute thrombosis in the coronary implantation of the Palmaz-Schatz endoprosthesis. Rev Esp Cardiol. 1994;47:747-53.

3.- ALTAVILLA D, DEODATO B, CAMPO GM, ARLOTTA M, MIANO M, SQUADRITO G, et al. A novel dual vitamin Elike antioxidant, inhibits activation of nuclear factor-kappaB and reduces the inflammatory response in myocardial ischemia-reperfusion injury. Cardiovasc Res. 2000;47:515-528.

4.- BALDI A, ABBATE A, BUSSANI R, PATTI G, MELFI R, AN-

GELINI A, et al. A poptosis and post-infaction left ventricular remodeling. J Mol Cell Cardiol. 2002; 34: 165-174.
5.- SIWIK DA, PAGANO PJ, COLUCCI WS. Oxidative stress regulates collagen síntesis and matriz metalloproteinase activity in cardiac fibroblast. Am J Physiol Cell Physiol. 2001; 280: C53-C60.

6.- FINKEL T. Signal transduction by reactive oxygen species in non-phagocitic cells. J Leukoc Biol. 1999; 65: 337-40.

7.- RETAMAL MA, CORTES CJ, REUSS L, BENNETT MV, SAEZ JC. S-nitrosylation and permeation through connexin 43 hemichannels in astrocytes: induction by oxidant stress and reversal by reducing agents. Proc Natl Acad Sci USA. 2006; 103: 4475-4480.

8.- SAEZ JC, RETAMAL MA, BASILIO D, BUKAUSKAS FF, BENNETT MV. Connexin-based gap junction hemichannels: gating mechanisms. Biochim Biophys Acta. 2005;1711:215224.

9.- KANG JJ, CHENG YW, FU WM. Studies on neuromuscular 
blockade by boldine in the mouse phrenic nerve-diaphragm. Jpn J Pharmacol. 1998;76:207-212.

10.-IVORRA M, ISSEKUTZ A, CORTIJOJ, MORCILLO E, BLAZQUEZ M, SANZ M, et al. Effect of boldine, secoboldine, and boldine methine on angiotensin II-induced neurtrophil recruitment in vivo. J Leukoc Biol.2005; 78: 696-704.

11.- ARISMENDI M, HA SÁNCHEZ, BK CASSELS, JC SÁEZ.

Boldina Bloquea los hemicanales pero no los canales de unión en hendidura en células HeLa transfectadas con conexina. Abstract Reunión Anual de la Sociedad de Fisiología de Chile, 2006.

12.- ALMEIDA ER, MELO A, XAVIER H. Toxicological evaluation of the hydro-alcohol extract of the dry leaves of Peumus boldus and boldine in rats. Phytother Res.2000; 14: 99-102.

13.- OCARANZA MP, GODOY I, JALIL JE, VARAS M, COLLANTES P, PINTO M, et al. Enalapril attenuates downregulation of Angiotensin-converting enzyme 2 in the late phase of ventricular dysfunction in myocardial infarcted rat. Hypertension. 2006;48:572-578.

14.- SHEU J, CHANG L, CHIANG C, SUN C, CHANG N, YOUSSEF A, et al. Impact of Diabetes on Cardiomyocyte Apoptosis and Connexin43 Gap Junction Integrity. Int Heart J. 2007; 48: 233-245.

15.- BIALIK S, GEENEN DL, SASSON IE, CHENG R, HORNER JW, EVANS SM, et al. Myocyte apoptosis during acute myocardial infarction in the mouse localizes to hypoxic regions but occurs independently of p53. J Clin Invest 1997; 100: 1363 1372.

16.- AMMARGUELLAT F, LAROUCHE I, SCHIFFRIN. Myocardial fibrosis in DOCA-Salt hypertensive rats. Circulation 2001;103:319-324.

17.- PETERSON J, LI H, DILLON L, BRYANT J. Evolution of matrix metalloprotease and tissue inhibitor expression during heart failure progression in the infarcted rat. Cardiovasc Res 2000; 46: 307-315.

18.- ELTZE M, GREBE T, MICHEL M, CZYBORRA P, ULLRICH B. Affinity profile at a1- and a2-adrenoceptor subtypes and in vitro cardiovascular actions of (+)-boldine. Eur J Pharmacol 2002; 443:151-168.

19.- O'BRIEN P, CARRASCO-POZO C, SPEISKY H. Boldine and its antioxidant or health-promoting properties. Chem Biol Interact. 2006; 159; 1-17.

20.- YOUN Y, KWON O, HAN E, SONG J, SHIN Y, LEE C. Protective effect of boldine on dopamine-induced membrane permeability transition in brain mitochondria and viability loss in PC12 cells. Biochem. Pharmacol. 2002; 63: 495-505.

21.- KONRATH E, SANTIN K, NASSIF M, LATINI A, HENRIQUES A, SALBEGO C. Antioxidant and pro-oxidant proper- ties of boldine on hippocampal slices exposed to oxygen-glucose deprivation in vitro. Neurotoxicology. 2008; 29:1136-40.

22.- FERNÁNDEZ J, LAGOS P, RIVERA P, ZAMORANO-PONCE E. Effect of boldo (Peumus boldus Molina) infusion on lipoperoxidation induced by cisplatin in mice liver. Phytother Res 2009; 23:1024-7.

23.- BACKHOUSE N,DELPORTE C, GIVERNAU M, CASSELS BK, VALENZUELA A, SPEISKY H. Anti-inflammatory and antipyretic effects of boldine. Agents Actions 1994; 42: 114 117.

24.- GOTTELAND M, JIMENEZ I, BRUNSER O, GUZMAN L, ROMERO S, CASSELS BK, et al. Protective effect of boldine in experimental colitis. Planta Med 1997; 63: 311-331.

25.- JANG YY, SONG JH, SHIN YK, HAN ES, LEE CS. Protective effect of boldine on oxidative mitochondrial damage in streptozotocin-induced diabetic rats. Pharmacol. Res 2000; 42: 361-371.

26.- SANTANAM N, PENUMETCHA M, SPEISKY H, PARTHASARATHY S. A novel alkaloid antioxidant, boldine and synthetic antioxidant, reduced form of RU486, inhibit the oxidation of LDL in vitro and atherosclerosis in vivo in LDLR-/mice. Atherosclerosis 2004; 173: 203-210.

27.- ASENCIO M, DELAQUERRIÉRE B, CASSELS BK, SPEISKY H, COMOY E, PROTAIS P. Biochemical and behavioral effects of boldine and glaucine on dopamine systems. Pharmacol Biochem Behav 1999; 62: 7-13.

28.- JENNINGS RB, SOMMERS HM, SMYTH GA, FLACK HA LINN H. Myocardial necrosis induced by temporary occlusion of a coronary artery in the dog. Arch Pathol. 1960; 70: 68-78.

29.- LEMASTERS JJ, BOND JM, CHACON E, HARPER IS, KAPLAN SH, OHATA H, et al. The $\mathrm{pH}$ paradox in ischemiareperfusion injury to cardiac myocytes. EXS 1996; 76: 99 -114.

30.- TANI M, NEELY JR. Role of intracellular Na in Ca2 overload and depressed recovery of ventricular function of reperfused ischemic rat hearts. Possible involvement of $\mathrm{H}-\mathrm{Na}$ and $\mathrm{Na}-\mathrm{Ca} 2$ exchange. Circ Res 1989; 65: 1045-1056.

31.- KUSUOKA H, PORTERFIELD JK, WEISMAN HF, WEISFELDT ML, MARBAN E. Pathophysiology and pathogenesis of stunned myocardium. Depressed Ca2 activation of contraction as a consequence of reperfusion- induced cellular calcium overload in ferret hearts. J Clin Invest 1987; 79: 950 -961.

32.- THOMPSON-GORMAN SL, ZWEIER JL. Evaluation of the role of xanthine oxidase in myocardial reperfusion injury. $\mathrm{J}$ Biol Chem. 1990; 265: 6656-6663.

33.- RADOMSKI MW, PALMER RM, MONCADA S. Endogenous nitric oxide inhibits human platelet adhesion to vascular endothelium. Lancet. 1987; 2: 1057-1058.

34.- WANG P, ZWEIER JL. Measurement of nitric oxide and pe- 
roxynitrite generation in the postischemic heart. Evidence for peroxynitritemediated reperfusion injury. J Biol Chem. 1996; 271: 29223-29230.

35.- VIAPPIANI S, NICOLESCU AC, HOLT A, SAWICKI G, CRAWFORD BD, LEON H, et al. Activation and modulation of $72 \mathrm{kDa}$ matrix metalloproteinase- 2 by peroxynitrite and glutathione. Biochem Pharmacol. 2009; 77: 826 - 834.

36.- OKAMOTO T, AKAIKE T, SAWA T, MIYAMOTO Y, VAN DER VLIET A, MAEDA H. Activation of matrix metalloproteinases by peroxynitrite-induced protein S-glutathiolation via disulfide S-oxide formation. J Biol Chem 2001; 276: 29596 - 29602.

37.- OVECHKIN AV, TYAGI N, RODRIGUEZ WE, HAYDEN MR, MOSHAL KS, TYAGI SC. Role of matrix metalloproteinase-9 in endothelial apoptosis in chronic heart failure in mice. J Appl Physiol. 2005; 99: 2398 -2405.

38.- MORIMOTO H, HIROSE M, TAKAHASHI M, KAWAGUCHI M, ISE H, KOLATTUKUDY P, et al. MCP-1 induces cardioprotection against ischaemia/reperfusion injury: role of reactive oxygen species. Cardiovasc Res 2008; 78: 554562.

39.- KOLATTUKUDY PE, QUACH T, BERGESE S, BRECKENRIDGE S, HENSLEY J, ALTSCHULD R, et al. Myocarditis induced by targeted expression of the MCP-1 gene in murine cardiac muscle. Am J Pathol 1998; 152: 101-111.

40.- ZHOU L, AZFER A, NIU J, GRAHAM S, CHOUDHURY M, ADAMSKI F, et al. Monocyte chemoattractant protein-1 induces a novel transcription factor that causes cardiac myocyte apoptosis and ventricular dysfunction. Circ. Res 2006; 98: 1177-1185. 